\title{
A Survey on Detecting Plant Diseases Detection and Proposal of a Solution using Recommendation System
}

\author{
Hanif Khan Pathan \\ CSE Department, Dr APJ Abdul Kalam University \\ Indore Dewas Bypass Road Indore, India
}

\author{
Dhanraj Verma, PhD \\ CSE Department, Dr APJ Abdul Kalam University \\ Indore Dewas Bypass Road INDORE, India
}

\begin{abstract}
Agriculture is the backbone of the Indian economy. A significant amount of people in India depends on agricultural income. But due to traditional methods of farming and dependency on nature, infection, and other different diseases, the production, and profit of crops are affected, results in poor quality and productivity. This paper is motivated to investigate the recent advancement in the agriculture based on computational technology. Therefore, recent technique of efficient and accurate plant disease detection using Machine Learning (ML) and image processing techniques has proposed to study. A survey has been carried out and the summary of conducted review has been reported. The reviewed literature is focused on categorizing the methods based on ML algorithms used. Additionally, the trends of utilization of ML techniques are also described. Using the concluded reviews and available facts we proposed a ML model for early disease prediction, and also proposed a recommendation system which provides relevant solution to deal with the disease is described. The different components of both the models and required functional aspects are also discussed. Finally, the paper provides the conclusion of the work carried out and future guidelines.
\end{abstract}

\section{Keywords}

Review, survey, plant disease detection and recommendation, machine learning algorithms, feature selection.

\section{INTRODUCTION}

India is an agricultural country, and act as the main source of livelihood. More than $80 \%$ of population has dependent on agriculture. Additionally in different areas of agriculture 52\% of the population is getting their employment. Its contribution is between 14 to $15 \%$ in Indian GDP. However, most of the farmers are totally dependent on agriculture [1]. Additionally, some of them are completely depend on seasonal crops. The production of crops is directly affecting the sustainability of the farmers of India. But due to rain, disease, and other influences most of the crops are ruined. Moreover, the bacteria, viruses, and fungus can also affect the crop yield [2]. Natural disasters are not manageable by us but we can reduce the impact.

In this context, the proposed work is motivated to explore and investigate the diseases in seasonal crops using ML and image processing technique. In recent years a significant advancement in agriculture using ML has been observed. Using ML techniques a number of applications are become easy to implement which can be beneficial for farmers by assisting in various involved processes in agriculture. This kind of technology is known as smart farming. In this presented work we are proposed to develop a ML model for detecting the infection and/or disease in crops, by analyzing the crop plant leafs. In literature, a number of techniques have existing that is claiming to provide accurate analysis of plant leaf images [3]. Among most of them are based on CNN (Convolutional neural networks) [4], SVM (support vector machine) [5] and image segmentation techniques [6].

The ML techniques are played an essential role in early disease detection in plants by analyzing the plant leaf images. Both kinds of techniques (i.e. supervised as well as unsupervised) can be used for this task [7]. Additionally, there are various other frameworks are also available that analyze the crop disease and recommend the solution to deal with the disease infection. Such kind of systems are useful in reduce the farmer's loss due to diseases. But these approaches are limited for some specific type of plants and crops. Therefore, in this work we are focused on finding more efficient and accurate methods. Thus in this paper we first involves a review of existing advancement for plant disease detection using ML and image processing techniques. In this review we categorize the contributions based on the employed ML algorithms. Further, the review is summarized to identify the required direction of study. In this context an outline of a solution has been provided. This model will be used to prepare design of the disease detection system.

\section{BACKGROUND}

An overview of the various common concepts which are frequently used for classifying and identifying the plant diseases is discussed in this part of paper.

\subsection{Image segmentation}

Image segmentation is the method of partitioning an image into multiple sets of pixels. The goal of segmentation is to simplify or transform the representation of an image to enhance the feature of the image. The aim of segmentation is to find a more meaningful and easier part of data for data analysis (classification and prediction). Image segmentation is used to locate the boundaries of objects in images. Additionally, it is the process of assigning a label to each pixel in an image according to certain characteristics. The result of segmentation is a set of segments representing the entire image or a set of contours. Each of the pixels in a region is similar in terms of some characteristic or property, i.e. color, intensity, or texture. An example of image segmentation is demonstrated in figure 1. 


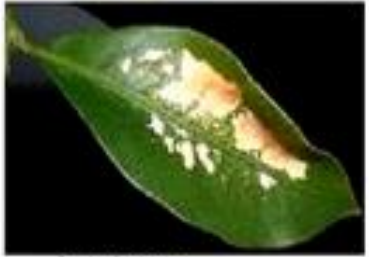

Inout image

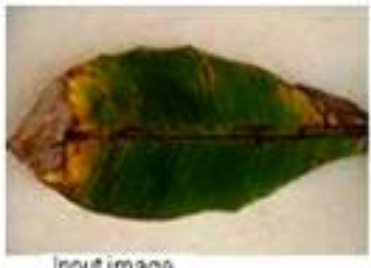

Input image

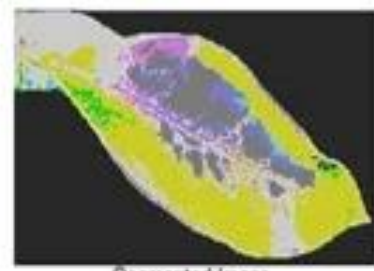

Segmented rage

Segmetted lmage

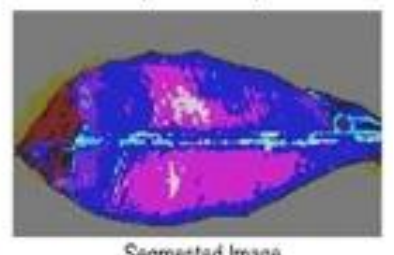

Figure 1 Example of Image Segmentation

\subsection{Image Classification \& CNN}

In different kinds of variants of Artificial Neural Network (ANN) the CNN is a special kind of architecture. It uses visual features. That is mainly designed for employing in image classification problems. For example, Facebook uses CNN for tagging, for users' photos. The main task of image classification is the acceptance of the input image and defines its class label. In this context the image is passed in a series of Convolutional, nonlinear, pooling layers and fully connected layers, then generates the output. The Convolution layer is the first layer. The image pixels are entered into it. The reading of the input matrix begins at the top left of the image. Next, it selects a smaller matrix here, called a filter. Then the filter produces convolution by moves along with the input image. The filter's task is to multiply its values by the original pixel values. All these multiplications are summed up and a number is obtained at the end. Finally, after processing the entire image, a matrix is obtained, which is smaller than the input matrix. Additionally, it consists of mixed nonlinear networks and pooling layers. The output of one layer becomes the input for the next layer, and so on. The nonlinear layer is added after each convolution operation. It is an activation function, which brings nonlinear property. The pooling layer is nonlinear, which works with the width and height of the input and do down-sampling. This means if some features have been identified in the previous process then complete image is not needed, thus only less detailed information required. After completion of a series of layers, it is required to include fully connected layers, which accept output of Convolutional networks. This layer used to the end of the network for producing results according to number of classes.

\subsection{Support Vector Machine (SVM)}

In ML research, SVM is an essential technique, which offers robust and accurate results with respect to other well-known machine learning algorithms. It is a sound technique for learn by example task, and suitable for higher-dimensional data. Fast training execution of the SVM algorithm is also available. The aim of SVM is to find the best classification function for binary. The concept of "best" classification function is measured geometrically. To classify a linear dataset, a linear classification function corresponding to a hyperplane $f(x)$ is created that passes through the middle of separable classes. Once function is determined, data instance $x_{n}$ can be classified simply applying the function $f\left(x_{n}\right)$. In these conditions $x_{n}$ belongs to the positive class when $f\left(x_{n}\right)>0$. Because there are various linear hyper planes is available using which the SVM guarantees to improve margin among classes. The margin is the space accessible between classes. The margin is corresponding to the shortest distance between the closest data points. This allows us to explore and maximize the margin, though an infinite number of hyper-planes.

\section{LITERATURE SURVEY}

This section provides understanding about the recent development and investigation on the plant disease detection and recommendation.

\subsection{Use of CNN}

In India, Agriculture plays an essential role because of the rapid growth of the population and the increased demand for food. One major effect on low crop yield is a disease caused by bacteria, viruses, and fungus. It can be prevented by using plant disease detection techniques. Machine learning methods can be used for disease identification. Mrs. U. Shruthi et al [8] present the stages of plant disease detection and comparative study on machine learning techniques. Authors observed that CNN gives high accuracy and detects more number of diseases of multiple crops. S. Sladojevic et al [10] is intended to develop a new plant disease recognition system by classification of leaf images using CNN. This system can recognize plant diseases of 13 types, and also difference between plant leaves. In order to implementing this model all the steps are described. They are gathering images to create a database. The developed system is using Berkley Vision and Learning Centre for training. The model provides the precision between $91 \%$ and $98 \%$ training and for validation provides an average of $96.3 \%$ precision. K. P. Ferentinos et al [12], developed CNN models to detect plant disease using leaves images in terms of healthy and infected. Training was performed with an open database. Among several models, the best performance success rate is found $99.53 \%$. The improved detection accuracy makes it a useful tool for early warning, and plant disease detection system.

E. Fujita et al [17] propose a new plant-disease detection system using 7,520 cucumber leaf images comprising of healthy leaves and infected by almost all types of diseases. The leaves were photographed on the requirement, is, each image must contain a leaf roughly at its center. Although half of the images used in this experiment were taken in bad conditions, a classification system based on CNN attained an average of $82.3 \%$ accuracy under the 4-fold cross-validation. K. Nagasubramanian et al [24] provide a 3D CNN mode to assimilate the hyper-spectral data. Author analyzes the model to generate physiological reports. They focus on economical important disease, which is a soil-borne fungal disease of soybean. Based on the imaging of stem images and 3D DCNN they got accuracy of $95.73 \%$ and F1 scores of 0.87 . Using a saliency map, they visualize the sensitive pixels and show the regions with disease symptoms. They also find the sensitive wavelengths used for classification in the near-infrared region, which is used spectral range for determining the health. The use of this model not only provides high accuracy but also provides physiological predictions. These predictions lend themselves for eventual use in precision agriculture and research.

Using PlantVillage dataset, which consist of four classes, a deep CNN is trained to severity analysis. The shallow networks and deep models tuned by transfer learning by G. Wang et al [26]. The deep VGG16 model is trained, which provide $90.4 \%$ accuracy. P. Sharma et al [27] investigates a solution by using the segmented image to train the $\mathrm{CNN}$ 
models. As compared to the F-CNN trained using full images, S-CNN trained using segmented images doubles in performance to $98.6 \%$ accuracy by the models with 10 diseases. By using a tomato plant and spot disease type, they show that the confidence of self-classification for the S-CNN improves on F-CNN. A. K. Rangarajan et al [15] studied tomato leaves images of 6 diseases and healthy class from the Plant-Village dataset. The two deep learning models namely AlexNet and VGG16 net are considered. The analysis is presented based on number of images and parameters such as mini-batch size, weight, bias, learning rate, accuracy and execution time.

\subsection{SVM \& ANN}

M. Islam et al [9] present an approach to integrate image processing and machine learning to diagnose diseases from leaf images. This method classifies diseases on potato plants from a publicly available plant image database. The segmentation approach and SVM demonstrate disease classification with an accuracy of $95 \%$. An increase in agroproducts affects the GDP of the nation. S. Iniyan et al [20] have a concise discussion with the detection of crop diseases using machine learning, especially with SVM and ANN. They have concluded a survey with the pros and cons of every method in context with input parameters. Crops can be infected by fungi, bacteria, and viruses and also by water and, temperature. M. A. Hussein et al [29] proposed a plant disease detection system. This model includes two phases, (1) knowledge bases that usage pre-processing techniques and used with SVM to train the model. (2) The trained classifier is used for detecting disease on plant leaf. Dataset contains 799 samples divided in $80 \%$ and $20 \%$, for training and testing. Three crops diseases are identified with the accuracy of $88.1 \%$. K. Golhani et al [14] reviews advanced Neural Network techniques to process hyper-spectral data, emphasis on plant disease. Firstly, provides a review of NN mechanism, types, models, and classifiers. Then they highlight the imaging and non-imaging data utilized for the detection purpose. The hybrid NN-hyper-spectral techniques are a powerful tool. The ratio of different spectral bands of disease spectra is termed as Spectral Disease Index (SDI). They introduce NN techniques for developing SDI and highlight trends and challenges.

\subsection{Other ML Techniques}

S. Ramesh et al [11] use Random Forest algorithm for recognizing healthy and diseased leaf. The implementation includes different phases like dataset creation, feature extraction, training, and classification. The datasets of plant leaves are trained using Random Forest to predict diseased and healthy leafs. Histogram of Oriented Gradient (HOG) is used for features extraction. X. Q. Yue et al [28] implemented an efficient, accurate, non-destructive, and simple technique to recognize maturity of strawberry. The maturity is divided into mature, nearly-mature and immature classes. The aim is to distinguish between mature and nearly-mature to help and improve profit of farmer. The images with different maturities of wavelengths using smart-phone are captured. The data is used to prepare recognition technique based on, multivariate linear, nonlinear and SoftMax regression. The multivariate nonlinear model produces $94 \%$ of highest accuracy.

\subsection{Segmentation}

V. Singh et al [13] presents an image clustering technique for detection of plant diseases using leaf. It includes a survey of different techniques of classification which will be used for this task. Image segmentation, is done using genetic algorithm. P. K. Sethy et al [30] was implemented a model to compute the plant disease severity in rice crop. The symptoms are used to get the seriousness of the disease and then suggest best technique to handle disease of rice plant. In rice plant disease are appear as a spot on the leaves. It is needed to analyze properly and on-time to avoid losses. The Fuzzy Logic with K-Means is used to compute the severity. This technique provides accuracy up to $86.35 \%$. C. K. Reddy et al [33] aim is to solve different diseases exposed by Different trees. So, a dataset was taken namely Wilt dataset that consists of diseased trees along with land cover fed to the algorithm to identify whether tree is diseased or not. The samples for the 'diseased trees' and 'other land cover' data comprises of numerical values related to the texture, generated by segmenting image and also segments contain spectral and texture information from the Quick bird multispectral image. A. M. Abdu et al [25] introduced a disease symptom clustering technique based on pathological pattern recognition on leaf images. The uniqueness of the technique is in the use of diseases pathological analogy, distinct homogeneous patterns for disease detection. The algorithm group images into symptomatic, necrotic, and blurred parts. The technique quantizes the disease lesion areas according to their analogy. This produces pattern of each leaf surface and features are extracted. The algorithm is combination of CIElab with deltaE $(\Delta \mathrm{E})$ color relativity and simple color space manipulation HSV. The results are acceptable, and can localizing and quantifying disease. It also provides opportunities for early detection of changes in plant growth, disease stage and severity.

\subsection{Reviews}

A. Lowe et al [22] explore the techniques developed for crop monitoring using imaging. The applications are outlined in both the scenarios in open farm as well as in glasshouse. Additionally, techniques are categorized into 'healthy and diseased with higher accuracy, early detection, and severity. The review usage the hyper-spectral imaging and briefed how these image are being utilized to predict disease. A summary is used for detecting stress including biotic and non-biotic. Researchers are exploring and investigating the plants resistance genes and trying to improve resistance in plant. Meanwhile, some of them developed detection and rating techniques for monitoring and predicting diseases. X. Yang et al [23] present the application of ML in resistance plant genes discovery and classification. Plants are affected by heterogeneous diseases through their leaves. Leaves are important for the fast-growing of the plant. To detect plant diseases fast techniques need to be adopted. S. S. Kumar et al [32] have done a survey on different plants disease and advance techniques to detect these diseases.

P. Thakur et al [31] provides a review and comparison of the different image processing methods for disease detection based on the features (i.e. color, texture, shape, and hybrid). These features are helpful in differentiating the type of plant disease infected region. The author's consideration is on diseases that are caused by pathogens. According to A. Sehgal et al [21] plants are concerned with various diseases. Different analysis created ID and scoring technique to monitor and examine the growth or quality. This review shows the use of $\mathrm{AI}$ in the plant issues detection. Optical sensors are useful in detection of diseased plants. The system consists of the different sensor, the platform, and data analysis based the decision making. M. T. Kuska et al [16] provides brief review on challenges and opportunity of their usage in plant disease detection. The Optical sensor techniques are the key 
component of plant phenol-typing. Authors address hyperspectral imaging techniques in determining plant parameters for specific sensors.

DNA-based and serological methods are the tools for diagnosing plant disease accurately. F. Martinelli et al [18] outlined techniques based on protein analysis and nucleic acid. The reviews main findings are: (1) sensors based on the analysis of host, provide results immediately and helpful in early detection; (2) biosensors based can also detect infection instantly; (3) remote sensing with spectroscopy allow high results. They also explain how different tools will be used in disease management. J. P. Shah et al [19] presents a survey of different image processing and ML techniques. They present various techniques but also discuss important concepts of image processing and ML to plant disease classification. They carry out a study of 19 papers, work on rice plant diseases and other plants and fruits. These criteria include the size of the image no. of diseases, preprocessing, segmentation, types of classifiers, the accuracy of classifiers, etc. they utilize survey and propose a design of detection and classification of the rice plant.

\section{REVIEW SUMMARY}

This section provides a summary of collected literature. Here a total of 22 research and review articles are collected for review of different approaches.

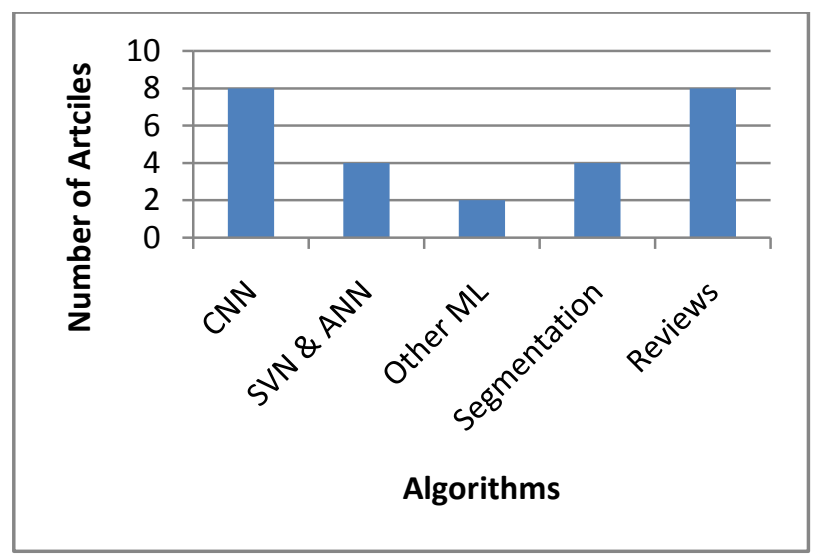

Figure 2 Algorithms trends

That is providing the guidelines for designing the proposed system. The figure 2 shows the algorithms frequently used for detecting the plant diseases for different crops, among them we found the $\mathrm{CNN}$ and other deep learning techniques are much frequently used now in these days. In addition to that, the SVM \& ANN are also used for predicting the diseases in crops using leaf images. In addition to that some of the authors who usages the biotic and non-biotic features are also used different other classification techniques for classifying the obtained or recovered features. Further one more significant technique observed namely segmentation techniques that are effective helps to early detection and recognition of plant growth monitoring, and level of disease.

\section{PROPOSED MODEL}

The proposed work is motivated to help the Indian farmers to early recognize the crop disease to reduce the financial loss. In this context we need an ML and image processing based technique for assisting the farmers. In this context an overview of the proposed methodology is given in figure 3 . The proposed model demonstrates the key components of the proposed Farmer Assistance Framework (FAF). In this section we are discussing the functional aspects of the FAF system.
The aim of this data centric model is to offer a low cost solution for Indian farmers. This model able to deal with the farmer's query related to crop disease based on plant leaf image. In addition, recommend the most suitable solution according to the disease identified on the crop. The FAF model involves two key prospects. First, the classification of disease, and second is the recommendation of appropriate solution. The system requires three modules in their design:

\subsection{Training}

The training data is an essential component of ML techniques. That directly impacts on the performance of the algorithms. The good quality and noise-free data provides higher performance as compared to noisy datasets. In order to prepare the knowledge base to need to collect the infected as well as healthy plants, name and disease, expert advice and possible solutions. Additionally, we organize all the information into an efficient retrieval mechanism. Because the system not only works for the classification of disease it also offers the solutions to prevent the losses. In this context, the image and text data is processed using the different techniques. First we normalize the data, and then the image and text features are computed. Here we need to explore the methods and techniques that can work with both the data types. Further we are enhancing the computed features to improve the indicators. These optimization techniques enhance the quality of features that are used for ML algorithms training and testing phases. The enhanced and structured data is keep preserved in the storage for utilizing in further phases.

\subsection{Learning and prediction of disease:}

That is the second phase of system, in these module, we implement supervised as well as unsupervised models to learn with the image features like SVM, CNN, and SOM. The supervised learning techniques needed learning examples to be learned, and then the trained model can be used for disease detection. On the other hand, the unsupervised learning techniques directly employable on the dataset and according to specific criteria the algorithm creates groups of data. But the supervised learning techniques are more accurate then unsupervised algorithms. Additionally, the unsupervised learning techniques are fast as compared to supervised learning techniques therefore in this work we will try to evaluate both kinds of models. In our FAF model the trained ML model is being used to solve the farmer's image query. The model takes image as input and recognizes the disease in a plant. That is the first phase consequence of the proposed FAF model.

\subsection{Recommending the solutions for disease}

The outcomes of the previous phase are used in this phase as input. The predicted disease based on farmer's image query is used as query to search relevant solution in knowledgebase. This module works as an Information Retrieval (IR) system. Thus using the text processing techniques is employed here to get most similar or less distance suitable solution for the recognized disease. The proposed system not only used for identifying the plant disease as well as it recommends the solution for the queried image by the farmer. In this paper we are just discussing the key concept and aim of the proposed FAF model. The complete design and development of the required model required keen investigation and a number of experimental analyses to formulate the effective, efficient and 
accurate model. Therefore the next section includes the future

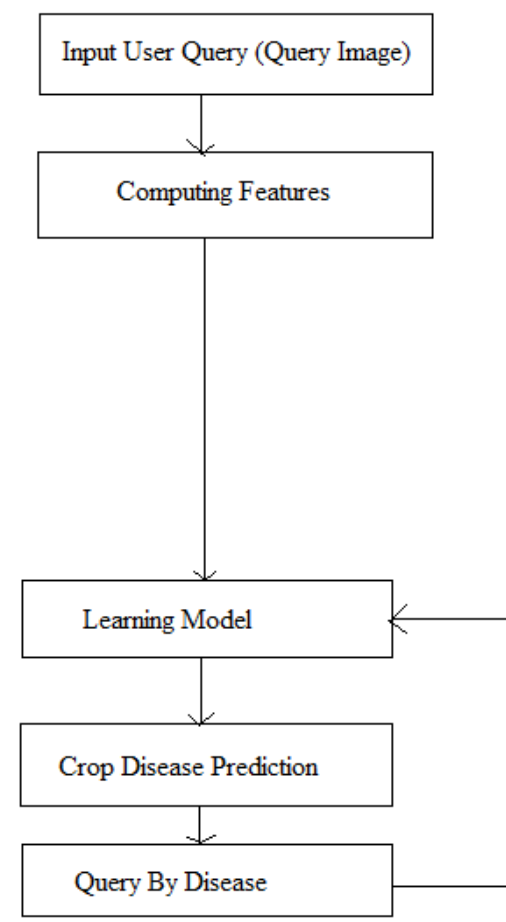

directions of the proposed study to be carry out.

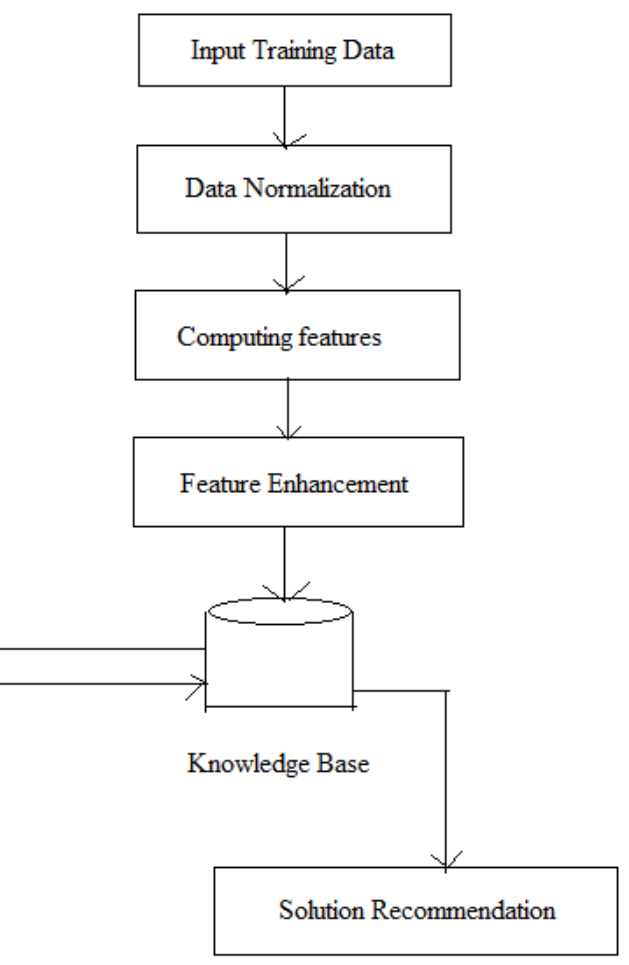

Figure 3 Proposed Farmer Assistance Technique Based on ML and Image Processing

\section{CONCLUSION}

The proposed work is motivated to explore the plant disease diagnosis techniques based on machine learning and image processing. Therefore we involved a study of the recently contributed techniques and enhancements. The review is categorized based on the ML algorithms used in the system design. Based on this study a summary of review has been presented. Further a FAF model is proposed for development and their essential components are also discussed. But the entire system modeling requires a layered plan for experimentation and component design. After successful implementation of the proposed FAF model provides the following fruitful outcomes:

1. A framework to accurately identify the disease in plants by analyzing the plant leafs. The model is needed to be design in such a way by which it becomes adoptive to incorporate more and new kinds of disease samples.

2. A unique system that also work blurred, lowresolution and noisy images to identify the disease. Farmer's can usages less resolution, poor quality cameras, or capture image in noisy surrounding therefore model needed to be robust against image quality.

3. A recommendation system to rectify the diseases in early stages. That becomes more helpful to farmers by providing the suitable solution for crop treatment to prevent the huge loss caused by the diseases.

In this context, the following task is proposed as objectives of for future work.
1. Investigate and design a dataset for the seasonal crops, disease, and their relevant solutions

2. Investigate the models that are able to process the images and accurately classify the healthy and crop infected by specific disease

3. Design a recommendation framework for suggesting the most appropriate solution to recover the plants from diseases

4. Carry out comparative performance study for justifying the proposed work.

\section{REFERENCES}

[1] "Agricultural http://www.hillagric.ac.in/aboutus /vc/vc_addresses/pdf/2017/08.12.2017-Agri.Edu.Day03.12. 2017.pdf

[2] P. Shrivastava, R. Kumar, "Soil salinity: A serious environmental issue and plant growth promoting bacteria as one of the tools for its alleviation", Sau. Jour. of Biol. Sci., 2015, 22, 123-131

[3] K. G. Liakos, P. Busato, D. Moshou, S. Pearson, D. Bochtis, "Machine Learning in Agriculture: A Review", Sensors 2018, 18, 2674; doi:10.3390/s18082674

[4] K. Kang, H. Li, J. Yan, X. Zeng, B. Yang, T. Xiao, C. Zhang, Z. Wang, R. Wang, X. Wang, W. Ouyang, "TCNN: Tubelets with Convolutional Neural Networks for Object Detection from Videos", Copyright 2017 IEEE

[5] M. Wang, Y. Wan, Z. Ye, X. Lai, "Remote sensing image classification based on the optimal support vector machine and modified binary coded ant colony optimization algorithm", Infor. Scie., 402, 2017, 50-68 
[6] G. Wang, W. Li , M. A. Zuluaga, R. Pratt, P. A. Patel, M. Aertsen, T. Doel, A. L. David, J. Deprest, S. Ourselin, T. Vercauteren, "Interactive Medical Image Segmentation Using Deep Learning With Image-Specific Fine Tuning", IEEE Trans. on Med. Ima., Vol. 37, No. 7, July 2018

[7] J. E. van Engelen, H. H. Hoos, "A survey on semisupervised learning", Mac. Lea., https://doi.org/10.1007/s10994-019-05855-6

[8] Mrs. U. Shruthi, Dr. V. Nagaveni, Dr. B. K Raghavendra, "A Review on Machine Learning Classification Techniques for Plant Disease Detection", 5th Inte. Conf. on Adv. Comp. \& Comm. Sys., 978-15386-9533-3/19/\$31.00 @2019 IEEE

[9] M. Islam, A. Dinh, K. Wahid, P. Bhowmik, "Detection of Potato Diseases Using Image Segmentation and Multiclass Support Vector Machine", 30th Cana. Conf. on Elec. \& Comp. Engg., 978-1-5090-5538-8/17/\$31.00 C2017 IEEE

[10] S. Sladojevic, M. Arsenovic, A. Anderla, D. Culibrk, D. Stefanovic, "Deep Neural Networks Based Recognition of Plant Diseases by Leaf Image Classification", Hind. Publ. Corp. Comp. Intel. \& Neuro. Vol. 2016, Art ID 3289801, 11 pages

[11] S. Ramesh, Mr. R. Hebbar, M. Niveditha, R. Pooja, N. P. Bhat, N. Shashank, Mr. P V Vinod, "Plant Disease Detection Using Machine Learning", 2018 Inter. Conf. on Des. Inn. for 3Cs Comp. Comm. Cont., 978-1-53867523-6/18/\$31.00 (02018 IEEE

[12] K. P. Ferentinos, "Deep learning models for plant disease detection and diagnosis", Comp. \& Elec. in Agri., 145, 2018, 311-318

[13] V. Singh, A. K. Misra, "Detection of plant leaf diseases using image segmentation and soft computing techniques", Info. Proce. in Agr. 4 (2017) 41-49

[14] K. Golhani, S. K. Balasundram, G. Vadamalai, B. Pradhan, "A review of neural networks in plant disease detection using hyper-spectral data", Info. Proc. in Agri. 5 (2018) 354-371

[15] A. K. Rangarajan, R. Purushothaman, A. Ramesh, "Tomato crop disease classification using pre-trained deep learning algorithm”, Proc. Comp. Sci. 133 (2018) $1040-1047$

[16] M. T. Kuska, A. K. Mahlein, "Aiming at decision making in plant disease protection and phenotyping by the use of optical sensors", Eur J Plant Pathol, https://doi.org/10.1007/s10658-018-1464-1

[17] E. Fujita, Y. Kawasaki, H. Uga, S. Kagiwada, H. Iyatomi, "Basic investigation on a robust and practical plant diagnostic system", 15th IEEE Inter. Conf. on Mach. Lear. \& Appl., 978-1-5090-6167-9/16 \$31.00 (c) 2016 IEEE

[18] F. Martinelli, R. Scalenghe, S. Davino, S. Panno, G. Scuderi, P. Ruisi, P. Villa, D. Stroppiana, M. Boschetti, L. R. Goulart, C. E. Davis, A. M. Dandekar, "Advanced methods of plant disease detection. A review", Agron. Sustain. Dev. (2015) 35:1-25, Springer-Verlag France 2014

[19] J. P. Shah, H. B. Prajapati, V. K. Dabhi, "A Survey on Detection and Classification of Rice Plant Diseases", IEEE inte. Conf. on 2016, 10, 1-8
[20] S. Iniyan, R. Jebakumar, P. Mangalraj, M. Mohit, A. Nanda, "Plant Disease Identification and Detection Using Support Vector Machines and Artificial Neural Networks", (c) Sprin. Natu. Sing. Pte Ltd. 2020

[21] A. Sehgal, S. Mathur, "Plant Disease Classification Using Soft Computing Supervised Machine Learning", IEEE Conf. Rec. \# 45616; ISBN: 978-1-7281-0167-5

[22] A. Lowe, N. Harrison, A. P. French, "Hyperspectral image analysis techniques for the detection and classification of the early onset of plant disease and stress", Pla. Meth. (2017) 13:80

[23] X. Yang, T. Guo, "Machine learning in plant disease research", Euro. Jour. of BioMe.1 Res., 2017 | Vol. 3 | Iss. 1

[24] K. Nagasubramanian, S. Jones, A. K. Singh, S. Sarkar, A. Singh, B. Ganapathysubramanian, "Plant disease identification using explainable 3D deep learning on hyper spectral images", Pla. Meth. (2019) 15:98

[25] A. M. Abdu, M. M. Mokji, U. U. Sheikh, "An Automatic Plant Disease Symptom Segmentation Concept Based on Pathological Analogy", 10th Con. \& Sys. Grad. Res. Coll., 2 - 3 August 2019, Shah Alam, Malaysia 978-17281-0754-7 IEEE

[26] G. Wang, Y. Sun, J. Wang, "Automatic Image-Based Plant Disease Severity Estimation Using Deep Learning", Hind. Comp. Intel. \& Neur. Vol. 2017, Article ID 2917536, 8 pages

[27] P. Sharma, Y. P. S. Berwal, W. Ghai, "Performance analysis of deep learning CNN models for disease detection in plants using image segmentation", Infor. Proc. in Agri.

[28] X. Q. Yue, Z. Y. Shang, J. Y. Yang, L. Huang, Y. Q. Wang, "A smart data-driven rapid method to recognize the strawberry maturity", Info. Proce. in Agri.

[29] M. A. Hussein, A. H. Abbas, "Plant Leaf Disease Detection Using Support Vector Machine", AlMustansiriyah Jour. of Sci., ISSN: 1814-635X, ISSN:2521-3520, Vol. 30, Iss. 1, 2019

[30] P. K. Sethy, B. Negi, N. K. Barpanda, S. K. Behera, A. K. Rath, "Measurement of Disease Severity of Rice Crop Using Machine Learning and Computational Intelligence", Cog. Scie. \& Arti. Inte., Spri. Bri. in Fore. \& Medi. Bioi., https://doi.org/10.1007/978-981-10-6698$6 \_1$

[31] P. Thakur, P. Aggarwal, M. Juneja, "Plant Disease Detection and Classification using Image Processing: A Review", Inter. Jour. of Rec. Rese. Asp. ISSN: 23497688, Vol. 4, Issue 3, Sept 2017, pp. 22-27

[32] S. S. Kumar, B. K. Raghavendra, "Diseases Detection of Various Plant Leaf Using Image Processing Techniques: A Review", 5th Inter. Confe. on Adv. Comp. \& Comm. Sys. 978-1-5386-9533-3/19/\$31.00 (C2019 IEEE

[33] C. K. Reddy, D. Hiranmayi, K. Eswaran, G. G. Likhita, B. Saritha, "Classification of Diseased Plants using Separation of Points by Planes", Inter. Jou. of Engg Tech. Sci. \& Rese., ISSN 2394 - 3386, Volume 4, Issue 9, Sept. 2017 